\title{
Steady-state kinetic analysis of mitochondrial respiratory enzymes from bovine heart mitochondria
}

\author{
Dayoung $\mathrm{Kim}^{1+}{ }^{\dagger}$, Eun $\mathrm{Ko}^{2+}$, Moonsung $\mathrm{Choi}^{3,4^{*}}$ and Sooim Shin ${ }^{1,2^{*}}$ (0)
}

\begin{abstract}
Mitochondria is a decisive organelle of cells that produces adenosine triphosphate (ATP) by the process of oxidative phosphorylation of the Krebs cycle and the electron transport chain. The electron transport chain system of mitochondria embodies multiple enzyme supercomplexes including complex I to $V$ which located in the inner membrane. Although the simple enzyme activity of some as-isolated complex has been studied so far, the steady-state kinetic analysis of each complex within the form of mitochondrial supercomplex has not been studied in depth. To this end, kinetic parameters of mitochondrial complex I-IV were determined using steady-kinetic analysis using corresponding substrates of them. Catalytic activity and binding affinity between substrates and enzymes were obtained by fitting the data to the Michaelis-Menten equation. Acquired kinetic parameters represented distinctive values depending on the complexes that can be interpreted by the characteristics of the enzymes including the distinction of substrates or the ratio of the enzyme itself under the supercomplex form. The indirect $k_{\text {cat }}$ of the mitochondrial enzymes were varied from 0.0609 to $0.334 \mathrm{~s}^{-1}$ in order of complex III, III, I, and IV and $K_{m}$ of substrates were also diverse from $5.1 \mu \mathrm{M}$ to $12.14 \mathrm{mM}$. This is the first attempt to get exact kinetic values that should provide profound information to evaluate the mitochondrial function practically in advance.
\end{abstract}

Keywords: Mitochondrial enzyme, Enzyme kinetics, Mitochondria supercomplex, Kinetic parameters

\section{Introduction}

The mitochondria play an important role in diverse aspects of cellular physiology, including production of adenosine triphosphate (ATP), regulation of oxidative stress, calcium homeostasis, and regulation of apoptosis [1]. In particular, ATP production occurs through the electron transport chain comprising four enzyme complexes (complex I-IV) and two electron carriers (ubiquinone and cytochrome c). Complex I, NADH: ubiquinone

\footnotetext{
*Correspondence: mschoi@seoultech.ac.kr; sooim.shin@jnu.ac.kr ${ }^{\dagger}$ Dayoung Kim and Eun Ko contributed equally to this work ${ }^{1}$ Interdisciplinary Program of Bioenergy and Biomaterials Graduate School, College of Engineering, Chonnam National University, Gwangju 61186, Republic of Korea

${ }^{3}$ Convergence Institute of Biomaterials and Bioengineering, Seoul National University of Science and Technology, Seoul 01811, South Korea Full list of author information is available at the end of the article
}

oxidoreductase, is an enzyme complex mediating the first step of the mitochondrial electron transport chain [2]. Complex I contains 45 subunits and has a molecular mass of about $1000 \mathrm{kDa}[2,3]$. It has an L-shaped structure comprising the membrane domain and peripheral domain, including the NADH binding site. Complex II, succinate dehydrogenase, catalyzes the conversion of succinate to fumarate in the mitochondrial matrix and reduces ubiquinone in the mitochondrial inner membrane. This dual action of complex II connects the citric acid cycle to the electron transport chain. In these steps, electrons are transferred from succinate to ubiquinone via the iron-sulfur cluster of FAD as an intermediate [4, 5]. Complex III, coenzyme Q: cytochrome c oxidoreductase, is an enzyme complex found in the inner mitochondrial membrane. It mediates the reduction of cytochrome $c$ by ubiquinol as a substrate. Mitochondrial complex III 
from bovine heart is dimeric, and each monomer comprises 11 different polypeptide subunits [5, 6]. Complex $\mathrm{IV}$, cytochrome $c$ oxidase, is the final enzyme of the electron transport chain and is located in the mitochondrial inner membrane. Complex IV oxidizes reduced cytochrome $c$ and transfers electrons to oxygen, thereby producing water.

Mitochondrial function has been used as a marker of cellular function in various research fields such as cancer, metabolic diseases, aging, and neurodegenerative diseases [7-11]. The dysfunction of mitochondria induces the progression of various diseases, and the status of diseases can be detected or analyzed by the mitochondrial function assay [12-14]. The most used methods for analyzing mitochondrial function include measurement of oxygen consumption, examination of ATP production, assessment of membrane permeability, and evaluation of its shape [15]. All of these are powerful and representative techniques to analyze the overall function of live and intact mitochondria. However, these techniques require sophisticated facilities and fresh mitochondrial fractions at the same time. Hence, assessment of the activity of mitochondrial complexes including that of the respiratory chain was proposed to overcome the difficulties of the requirement of high-end techniques and demand for fresh mitochondria [16]. However, the activity assay of mitochondrial enzymes is also limited because it analyzes simple activity of enzymes consuming substrates without in-depth analysis of enzyme kinetic parameters.

To this end, steady-state kinetic parameters of mitochondrial enzymes from bovine heart mitochondria were determined within the composition of respiratory supercomplexes because each enzyme modulates specific reactions by consuming specific substrates. This method would reveal the kinetics of assembled enzyme complexes under physiological conditions, unlike the methods used in previous studies, which established the kinetics of isolated enzyme complexes. Determining the steady-state kinetic parameters of an enzyme is useful because the results would reveal the overall reaction or function of the enzyme, from binding of the substrate to the production of the final product.

\section{Materials and methods Reagents}

All of the reagents are purchased from Sigma Aldrich (MO, USA).

\section{Activity assay of mitochondrial complex I (NADH: Ubiquinone oxidoreductase)}

Isolated bovine heart mitochondria were purchased from Cayman Chemical (MI, USA). The activity of complex I was monitored by using the spectral property of oxidized NADH at $340 \mathrm{~nm}$ with an extinction coefficient (ع) of $6.2 \mathrm{mmol}^{-1} \mathrm{~cm}^{-1}$ as previously described [16]. The activity of complex I was measured using ultrapure distilled water, $50 \mathrm{mM}$ potassium phosphate buffer $(\mathrm{pH}$ $7.5), 3 \mathrm{mg} / \mathrm{mL}$ of bovine serum albumin, $300 \mu \mathrm{M}$ potassium cyanide $(\mathrm{KCN})$, and different concentrations of NADH $(2.5,5,10,25,50,75$, and $100 \mu \mathrm{M})$ using $3 \mu \mathrm{g}$ of bovine heart mitochondria. After $10 \mathrm{~min}$ of incubation at $37^{\circ} \mathrm{C}, 60 \mu \mathrm{M}$ of ubiquinone was added, and the change in absorption at $340 \mathrm{~nm}$ was measured for $600 \mathrm{~s}$ every $10 \mathrm{~s}$ using an S-3100 spectrophotometer (Scinco, Seoul, Republic of Korea). A parallel assay was carried out using the same conditions by using a fixed concentration of NADH $(100 \mu \mathrm{M})$ and different ubiquinone concentrations of $5,10,20,30,40,50,60$, and $70 \mu \mathrm{M}$.

\section{Activity assay of mitochondrial complex II (Succinate dehydrogenase)}

The activity of complex II was monitored using DCPIP (dichlorophenolindophenol) as a coupling reactant at $600 \mathrm{~nm}$ with an extinction coefficient $(\varepsilon)$ of $19.1 \mathrm{mmol}^{-1} \mathrm{~cm}^{-1}$ as previously described [16]. The activity of complex II was measured in ultrapure distilled water, $25 \mathrm{mM}$ potassium phosphate buffer ( $\mathrm{pH} 7.5$ ), $1 \mathrm{mg} /$ $\mathrm{mL}$ of BSA, $300 \mu \mathrm{M} \mathrm{KCN}, 80 \mu \mathrm{M}$ DCPIP, and different concentrations of succinate $(2.5,5,10,20,30,40,60$, and $80 \mathrm{mM}$ ) using $3 \mu \mathrm{g}$ of bovine heart mitochondria. After 10 min of incubation at $37^{\circ} \mathrm{C}, 50 \mu \mathrm{M}$ of decylubiquinone was added and the change in absorption at $600 \mathrm{~nm}$ was measured for $600 \mathrm{~s}$ every $10 \mathrm{~s}$ using an S-3100 spectrophotometer (Scinco). A parallel assay was carried out at the same conditions using a fixed concentration of $80 \mathrm{mM}$ of succinate and different concentrations of 5,10 , $20,30,40,50$, and $60 \mu \mathrm{M}$ of decylubiquinone.

\section{Activity assay of mitochondrial complex III (coenzyme Q:cytochrome c oxidoreductase)}

The activity of complex III was measured by using the spectral property of reduced cytochrome $c$ at $550 \mathrm{~nm}$ with an extinction coefficient $(\varepsilon)$ of $18.5 \mathrm{mmol}^{-1} \mathrm{~cm}^{-1}$ as previously described [16]. The activity of complex III was measured in ultrapure distilled water, $25 \mathrm{mM}$ potassium phosphate buffer ( $\mathrm{pH} 7.5), 500 \mu \mathrm{M} \mathrm{KCN}, 0.025 \%(\mathrm{v} / \mathrm{v})$ Tween-20, $100 \mu \mathrm{M}$ EDTA (ethylenediaminetetracetic acid), and $75 \mu \mathrm{M}$ oxidized cytochrome $c$ using $3 \mu \mathrm{g}$ of bovine heart mitochondria. After 10 min of incubation at $37{ }^{\circ} \mathrm{C}$, decylubiquinol of different concentrations $(2.5,5$, $7,10,12.5,18.5$ and $25 \mu \mathrm{M})$ was added, and the change in absorption at $550 \mathrm{~nm}$ was measured for $600 \mathrm{~s}$ every $10 \mathrm{~s}$ using an S-3100 spectrophotometer (Scinco). A parallel assay was carried out at the same conditions using a fixed concentration of $25 \mu \mathrm{M}$ of decylubiquinol and different 
concentrations of $5,10,20,30,40,50$, and $60 \mu \mathrm{M}$ of oxidized cytochrome $c$.

\section{Activity assay of mitochondrial complex IV (Cytochrome c oxidase)}

The activity of complex IV was measured by oxidation of reduced cytochrome $c$ using the spectral property of reduced cytochrome $c$ at $550 \mathrm{~nm}$ with an extinction coefficient $(\varepsilon)$ of $18.5 \mathrm{mmol}^{-1} \mathrm{~cm}^{-1}$ as previously described [16]. The activity of complex IV was measured in ultrapure distilled water, $50 \mathrm{mM}$ potassium phosphate buffer ( $\mathrm{pH} 7.0$ ), and different concentrations of reduced cytochrome $c(10,30,50,70,90$ and $120 \mu \mathrm{M})$. After $10 \mathrm{~min}$ of incubation at $37^{\circ} \mathrm{C}, 3 \mu \mathrm{g}$ of bovine heart mitochondria were added and the change in absorption was measured at $550 \mathrm{~nm}$ for $600 \mathrm{~s}$ every $10 \mathrm{~s}$ using an S-3100 spectrophotometer (Scinco).

\section{Kinetic analysis of mitochondrial complexes}

Based on the results of the absorption spectra obtained by the activity assay, the initial rate was measured from the linear portion of the reaction progress curve and fit according to the standard Michaelis-Menten equation (Eq. 1) to obtain the $V_{\max }$ and $K_{m}$ values. In Eq. 1, $V_{0}$ represents the initial rate, $V_{\max }$ represents the maximum reaction rate achieved by the system, and $K_{m}$ represents the concentration of the substrate at which the reaction rate is half of $V_{\max }$.

$$
V_{0}=V_{\max }[S] /\left(K_{m}+[S]\right)
$$

Indirect turnover rate of $k_{\text {cat }}$ of each enzyme was determined based on the Eq. 2. In Eq. 2, $V_{\text {max }}$ represents the maximum reaction rate achieved by the system, $k_{\text {cat }}$ represents the turnover number, and $\left[E_{t}\right]$ represents enzyme concentration applied as the ratio of each enzymes..

$$
V_{\max }=k_{c a t}\left[E_{t}\right]
$$

Analysis was conducted using the GraphPad Prism 8.0.1 software.

\section{Results and discussion}

The analysis of enzyme kinetics from the initial rate $\left(V_{0}\right)$ of reaction provides information that describes the function of enzymes from substrate binding to product formation, depending on the substrate concentration of each complex. To analyze the kinetic parameters of each enzyme by determining $V_{0}$, the enzyme activities of complexes I to IV were monitored by varying the concentrations of the specific substrates for each enzyme (Fig. 1). Given that every enzyme possesses a specific $k_{\text {cat }}$, the reaction was monitored by varying concentration of both substrates; therefore, high accuracy of measurements was obtained regardless of the variation in the substrates. Although the kinetic assay was conducted for individual enzymes within the whole mitochondrion, the reaction progress curves were in the shape of a single exponential, and all the progress curves of the enzyme complexes were saturated after a certain number of seconds, resulting in the plateau of a linear line within the last portion of the graph. From the graph, $V_{0}$ of the enzyme was measured at every concentrations of the substrates and were fitted to the Michaelis-Menten equation (Eq. 1) to obtain $V_{\text {max }}$ and $K_{m}$ values (Fig. 2 and Table 1 ). The results of the kinetic analysis indicate that each complex within the mitochondria exhibits specific kinetic property as shown by the characteristic $V_{\max }$ and $K_{m}$ values.

Complex IV exhibited a $V_{\text {max }}$ of $1.4380 \pm 0.4977 \mu \mathrm{M} \mathrm{s}^{-1}$, which was 4- to 8-fold faster than that of the other enzymes of complex I, II, and III. The other enzymes exhibited similar $V_{\text {max }}$, and the reaction rate was the highest for complex II followed by complex I and III (Table 1). From previous studies, it is known that enzymes forming respiratory supercomplexes possess specific ratios of complex I-IV, according to the origin of the organ and host organism [17]. The previous research identified the ratio of bovine heart mitochondrial complex I:II:III:IV as $1.1 \pm 0.2: 1.3 \pm 0.1: 3: 6.7 \pm 0.8$ [18]. Therefore, the indirect turnover rate of $k_{c a t}$ for each enzyme can be decided by dividing the $V_{\max }$ with the ratio of each enzyme within a single unit of mitochondria, based on Eq. 2 (Table 1). The value of indirect $k_{\text {cat }}$ for each complex was similar, except that for complex III $\left(0.06 \mathrm{~s}^{-1}\right)$, which was relatively low. Various values were also obtained for the affinity with substrates, $K_{m}$, depending on each enzyme. For complex II, the coupling reactant of DCPIP was applied for monitoring the reduction of decylubiquinone by succinate. Thus, the monitored progress curve portrayed the reduction of DCPIP, which was coupled with the activity of complex II. Therefore, the unit of calculated kinetic values of $K_{m}$ was shown by the concentration unit of DCPIP. To verify the $K_{m}$ of succinate as an exact varied substrate, the reaction ratio of $20 \mathrm{mM}: 50 \mu \mathrm{M}: 80 \mu \mathrm{M}$ for succinate, decylubiquinone, and DCPIP was applied [16]. The exact $K_{m}$ values of each complex with respect to their substrates are listed in Table 1.

The definition of parameters allows for the interpretation of distinct kinetic properties of mitochondrial complexes. For complex I, the calculated $V_{\text {max }}$ by varying both ubiquinone and $\mathrm{NADH}$ in separate reactions was similar at around $0.3 \mu \mathrm{M} \mathrm{s}^{-1}$, and the indirect $k_{\text {cat }}$ of the reactions was similar to that of other enzymes. However, the $K_{m}$ values of NADH and ubiquinone were $11.2397 \mu \mathrm{M}$ and $12.5676 \mu \mathrm{M}$, respectively, which were similar to those of the substrates of complex II and III and relatively lower than those of reduced cytochrome 

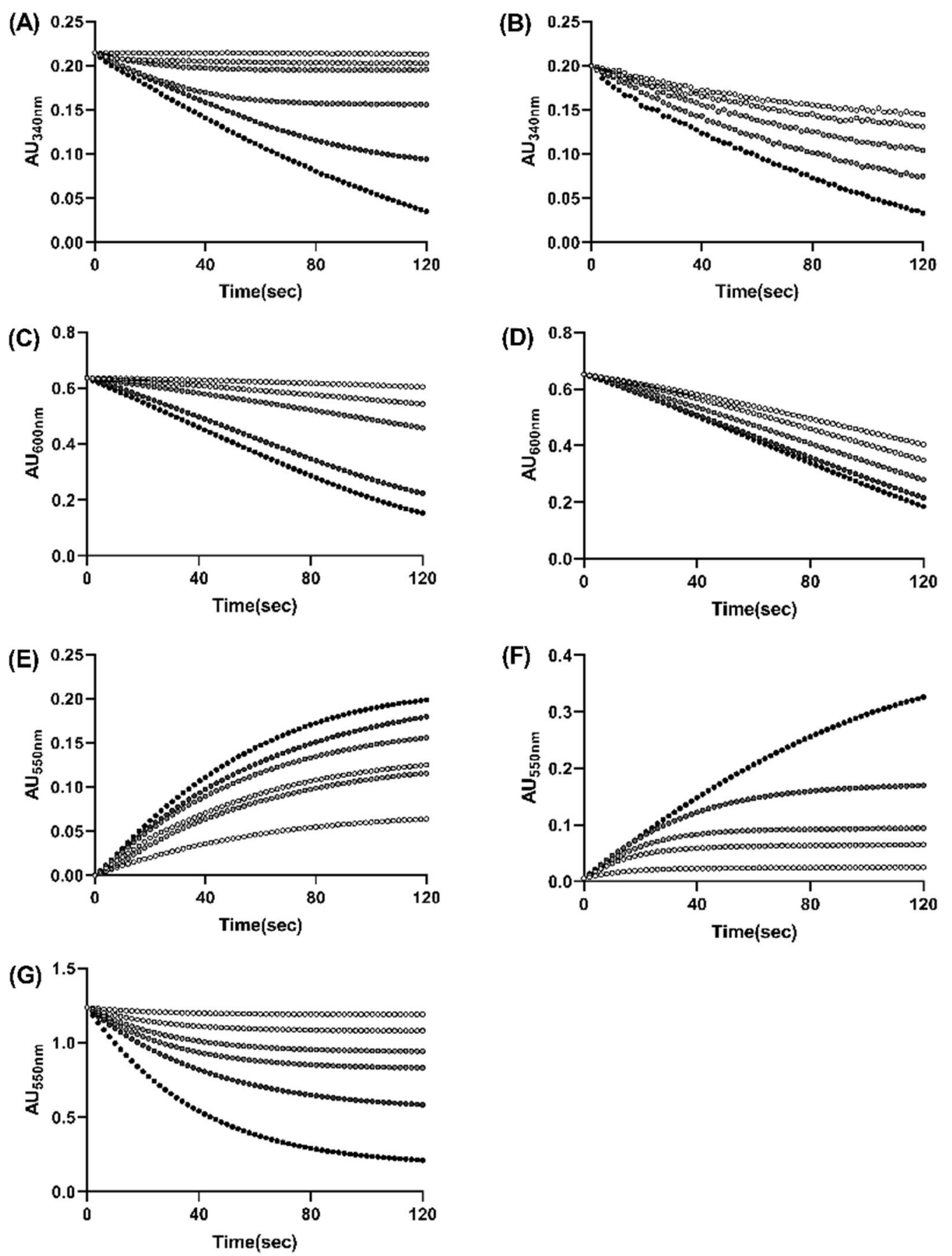

Fig. 1 Exemplar progression curve of mitochondrial enzyme activity. A Activity of NADH: ubiquinone oxidoreductase (complex I) with varying concentrations of $\mathrm{NADH}$, B activity of $\mathrm{NADH}$ : ubiquinone oxidoreductase (complex I) with varying concentrations of ubiquinone. $\mathbf{C}$ Activity of succinate dehydrogenase (complex II) with varying concentrations of succinate. D Activity of succinate dehydrogenase (complex II) with varying concentrations of decylubiquinone. $\mathbf{E}$ Activity of coenzyme Q: cytochrome c oxidoreductase with varying concentrations of decylubiquinol. $\mathbf{F}$ Activity of coenzyme Q: cytochrome c oxidoreductase with varying concentrations of oxidized cytochrome $c$. $\mathbf{G}$ Activity of cytochrome $c$ oxidase (complex IV) with varying concentrations of reduced cytochrome c. Deeper color of dots represents the higher concentrations of substrate for each graph 

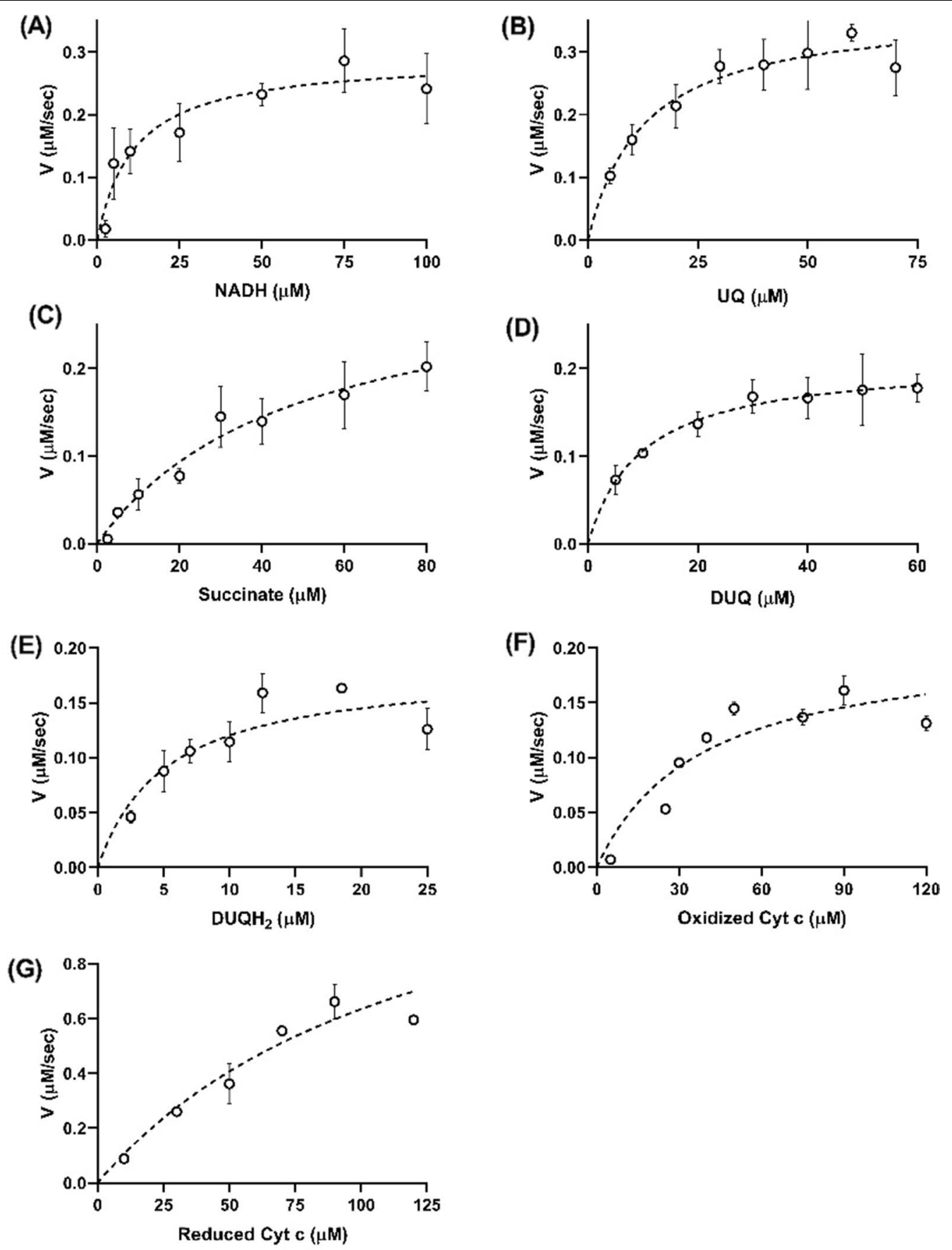

Fig. 2 Mitochondrial enzyme activity fitted to the Michaelis-Menten equation. A Activity of NADH: ubiquinone oxidoreductase (complex I) with varying concentrations of $\mathrm{NADH}$, B Activity of $\mathrm{NADH}$ : ubiquinone oxidoreductase (complex I) with varying concentrations of ubiquinone. C Activity of succinate dehydrogenase (complex II) with varying concentrations of succinate. D Activity of succinate dehydrogenase (complex II) with varying concentrations of decylubiquinone. E Activity of coenzyme Q: cytochrome c oxidoreductase with varying concentrations of decylubiquinol. $\mathbf{F}$ Activity of coenzyme Q: cytochrome $c$ oxidoreductase with varying concentrations of oxidized cytochrome $c$. G Activity of cytochrome $c$ oxidase (complex IV) with varying concentrations of reduced cytochrome $c$. Results represent mean and standard error for duplicated or triplicated experiments 
Table 1 Kinetic parameters of mitochondrial complexes

\begin{tabular}{|c|c|c|c|c|c|c|}
\hline & Varied substrates & $V_{\max }$ & $V_{\text {max }}$ unit & $K_{m}$ & $K_{m}$ unit & Indirect $k_{\text {cat }}\left(\mathrm{s}^{-1}\right)$ \\
\hline \multirow[t]{2}{*}{ NADH: Ubiquinone oxidoreductase } & $\mathrm{NADH}$ & $0.2909 \pm 0.0405$ & $\mu M \cdot s^{-1}$ & $11.2397 \pm 5.7066$ & $\mu \mathrm{M}$ & $0.2644 \pm 0.0368^{*}$ \\
\hline & UQ & $\begin{array}{l}0.3667 \\
\left(95 \% \mathrm{Cl}_{0} 0.3048-0.4640\right)\end{array}$ & & $\begin{array}{l}12.5676 \\
(95 \% \mathrm{Cl} \\
5.9611-25.9138)\end{array}$ & & $\begin{array}{l}0.3334^{*} \\
(95 \% \mathrm{Cl} 0.2771-0.4218)\end{array}$ \\
\hline \multirow[t]{4}{*}{ Succinate dehydrogenase } & Succinate & $0.3197 \pm 0.0759$ & & $48.5691 \pm 22.5309^{a}$ & & $0.2459 \pm 0.0584^{*}$ \\
\hline & & & & $12.1423 \pm 5.6327^{\beta, *}$ & $\mathrm{mM}$ & \\
\hline & DUQ & $\begin{array}{l}0.2095 \\
(95 \% \mathrm{Cl} 0.1731-0.2666)\end{array}$ & & $\begin{array}{l}9.7331^{a} \\
(95 \% \mathrm{Cl} 4.2749-21.0149)\end{array}$ & $\mu \mathrm{M}$ & $\begin{array}{l}0.1612^{*} \\
\left({ }^{95 \% C 1} 0.1332-0.2051\right)\end{array}$ \\
\hline & & & & $\begin{array}{l}6.0832^{\gamma_{i}^{*}} \\
\left(95 \% \mathrm{Cl}^{2} 2.6718-13.1343\right)\end{array}$ & & \\
\hline \multirow{2}{*}{$\begin{array}{l}\text { Coenzyme Q: cytochrome c oxidore- } \\
\text { ductase }\end{array}$} & $\mathrm{DUQH}_{2}$ & $0.1827 \pm 0.0287$ & & $5.1969 \pm 2.4045$ & & $0.0609 \pm 0.0096^{*}$ \\
\hline & Oxidized Cytc & $\begin{array}{l}0.2071 \\
(95 \% \mathrm{Cl} 0.1601-0.2893)\end{array}$ & & $\begin{array}{l}37.8970 \\
(95 \% \mathrm{Cl} 17.9899-79.0282)\end{array}$ & & $\begin{array}{l}0.0690^{*} \\
\left({ }^{95 \%} \mathrm{Cl} 0.0534-0.0964\right)\end{array}$ \\
\hline Cytochrome c oxidase & Reduced Cytc & $1.4380 \pm 0.4977$ & & $126.7201 \pm 69.9096$ & & $0.2146 \pm 0.0743^{*}$ \\
\hline
\end{tabular}

${ }^{*}$ Theoretically calculated value, ${ }^{a} K_{m}$ of DCPIP, ${ }^{\beta} K_{m}$ of Succinate, ${ }^{\gamma} K_{m}$ of Decylubiquinone

$c$ for complex IV $(126.7201 \mu \mathrm{M})$. This relatively low $K_{m}$ value might have been observed due to the characteristics of the substrates, ubiquinone and NADH. It is known that $V_{\text {max }}$ depends on the structure and concentration of the enzyme, whereas $K_{m}$ is independent of the enzyme concentration. Unlike complex IV, the activity assay of complex I, II, and III involved the utilization of quinone, quinol, or small-sized compounds as the substrate, which can easily be moved through the membrane. This might have facilitated the binding of additional small-sized substrate to the enzyme by reconstructing binding site of enzyme for easier substrate binding and low $K_{m}$ resulted from that. The calculated values of $V_{\max }$ and $K_{m}$ were smaller than those determined in previous results by using isolated complex I from bovine heart mitochondria [1] which is the only kinetic study for using isolated complexes. These results might also result from the difference of the system between isolated complex I and complex I in supercomplexes in which possess different environments around the enzymes. For complex II, a $K_{m}$ value of $6.0832 \mu \mathrm{M}$ for decylubiquinone, which is distinct from complex I despite of its activity, was also obtained using the quinone compound. This might have been a result of the specific structure of succinate dehydrogenase, the difference between decylubiquinone and ubiquinone, or the characteristics of its reaction that included a coupling reaction with DCPIP. For $V_{\max }$ or indirect $k_{c a t}$ although the values calculated by varying decylubiquinone were relatively lower than those calculated by varying succinate, they were similar within the error range. For complex III, relatively lower $V_{\max }$ and $K_{m}$ values compared to those of other enzymes were observed when they were measured by varying the concentrations of decylubiquinol. The indirect $k_{c a t}$ value of complex III was lower than that of other enzymes. Therefore, it is presumable that this resulted from the structure of coenzyme Q: cytochrome c oxidoreductase or the ratio of the enzymes among the supercomplexes, because $V_{\max }$ depends on the enzyme structure and concentration. A relatively high $K_{m}$ value of $37.8970 \mu \mathrm{M}$, which is similar to that of reduced cytochrome $c$ for complex IV, was obtained for the oxidized cytochrome $c$ for complex III. This indicated that the $K_{m}$ value of the substrate larger in size than quinone, quinol, or other smaller compounds is getting higher. Complex IV exhibited a very high $V_{\max }$ value of $1.4380 \mu \mathrm{M} \cdot \mathrm{s}^{-1}$ compared to the $V_{\max }$ values of other complexes, although the $k_{\text {cat }}$ value of $0.2146 \mathrm{~s}^{-1}$ for complex IV was similar to $k_{\text {cat }}$ values of 0.1612 and $0.3334 \mathrm{~s}^{-1}$ for complex I and II, respectively. Therefore, it can be concluded that the high concentrations represented by the high ratios of complex IV in bovine heart mitochondrial supercomplexes cause elevated $V_{\max }$. The results of this study reveal the kinetic parameters of mitochondrial respiratory complexes isolated from bovine heart mitochondria. Although these parameters are interpreted without considering the system environment, such as temperature, $\mathrm{pH}$, and concentrations of salt, it is important that the standard kinetic parameters were determined without the form of the isolated complex. Paradoxically, our results cannot represent the exact kinetic parameters of enzymes since whole mitochondrial fraction for the assay was utilized. However, we applied the ratio of the enzyme complexes to present the indirect $k_{c a t}$ for the understanding of enzyme function within the whole mitochondrial fraction. In addition, bovine heart mitochondria that is usually less-applied to the mitochondrial study, was used for the study rather than that in rodent animal models. It may decrease the utilizing potent of our kinetic values but the comparison of the values from 
bovine heart mitochondria to those from rodent animal models will be studied in further. Nonetheless, a kinetic analysis was carried out by varying the substrates of the enzymes, thus, increasing the reliability of the results. Although previous results have determined the kinetic values of enzymes using isolated complexes by varying both substrates, the present study is the first to describe the kinetic properties of mitochondrial enzymes in their original form in the mitochondrial supercomplex $[1,4]$. Furthermore, our results would be helpful to identify the functional state of mitochondrial enzymes in detail such as a change in substrate binding and further alterations in enzyme function by relatively simple methods compared to the previously described techniques. The main points of our methodological approach are not the replacement of the current techniques of mitochondrial assay such as oxygen consumption or structure analyzer, but to present deeper analysis within the enzyme kinetics level and to down the equipment-barrier for mitochondrial function assay by applying the relatively simple facility of a spectrophotometer. The determined kinetic parameters by our approach would be useful for deeper analysis of enzyme dysfunction-related malfunctioning of mitochondria and the occurrence of various diseases and can be applied to various fields of studies, including identifying the exact structure and composition of mitochondria and analyzing the effects of acute exposure of certain toxins on mitochondrial function.

\section{Acknowledgements}

Not applicable.

\section{Authors' contributions}

SS and MC conceived and designed the project, DK acquired the data, EK and SS analyzed and interpreted the data, DK, EK, and SS wrote the original draft, EK, SS, and MC edited and reviewed the manuscript, and SS and MC supervised the research. All authors read and approved the final manuscript.

\section{Funding}

This work was supported by the National Research Foundation of Korea [Grant Number 2020R1A2C1101110].

\section{Availability of data and materials}

The datasets used and/or analysed during the current study are available from the corresponding author on reasonable request.

\section{Declarations}

\section{Competing interests}

The authors declare that they have no competing interests.

\section{Author details}

${ }^{1}$ Interdisciplinary Program of Bioenergy and Biomaterials Graduate School, College of Engineering, Chonnam National University, Gwangju 61186, Republic of Korea. ${ }^{2}$ Department of Biotechnology and Bioengineering, College of Engineering, Chonnam National University, 77 Yongbong-ro, Buk-gu, Gwangju 61186, Republic of Korea. ${ }^{3}$ Convergence Institute of Biomaterials and Bioengineering, Seoul National University of Science and Technology, Seoul 01811 , South Korea. ${ }^{4}$ Department of Optometry, College of Energy and Biotechnology, Seoul National University of Science and Technology, Seoul 01811, Republic of Korea.

Received: 25 May 2021 Accepted: 15 July 2021

Published online: 23 July 2021

\section{References}

1. Nakashima Y, Shinzawa-Itoh K, Watanabe K, Naoki K, Hano N, Yoshikawa S (2002) Steady-state kinetics of NADH: coenzyme Q oxidoreductase isolated from bovine heart mitochondria. J Bioenerg Biomembr 34(1):11-19

2. Brandt U, Okun JG (1997) Role of deprotonation events in ubihydroquinone: cytochrome c oxidoreductase from bovine heart and yeast mitochondria. Biochemistry 36(37):11234-11240

3. Lenaz G, Fato R, Genova ML, Bergamini C, Bianchi C, Biondi A (2006) Mitochondrial Complex I: structural and functional aspects. BBA-Bioenergetics 1757(9-10):1406-1420

4. Jones AJ, Hirst J (2013) A spectrophotometric coupled enzyme assay to measure the activity of succinate dehydrogenase. Anal Biochem 442(1):19-23

5. Iwata S, Lee JW, Okada K, Lee JK, Iwata M, Rasmussen B, LinkTA, Ramaswamy S, Jap BK (1998) Complete structure of the 11-subunit bovine mitochondrial cytochrome bc1 complex. Science 281(5373):64-71

6. Chandel NS (2010) Mitochondrial complex III: an essential component of universal oxygen sensing machinery? Resp Physiol Neurobi 174(3):175-181

7. Modica-Napolitano JS, Singh KK (2004) Mitochondrial dysfunction in cancer. Mitochondrion 4(5-6):755-762

8. de Moura MB, dos Santos LS, Van Houten B (2010) Mitochondrial dysfunction in neurodegenerative diseases and cancer. Environ Mol Mutagen 51(5):391-405

9. SINGH K K, (2004) Mitochondrial dysfunction is a common phenotype in aging and cancer. Ann Ny Acad Sci 1019(1):260-264

10. Orth M, Schapira A (2001) Mitochondria and degenerative disorders. Am J Med Genet 106(1):27-36

11. Johannsen DL, Ravussin E (2009) The role of mitochondria in health and disease. Curr Opin Pharmacol 9(6):780-786

12. Hsu C-C, Tseng L-M, Lee H-C (2016) Role of mitochondrial dysfunction in cancer progression. Exp Biol Med 241(12):1281-1295

13. Bournat JC, Brown CW (2010) Mitochondrial dysfunction in obesity. Curr Opin Endocrinol 17(5):446

14. Bernardi P, Krauskopf A, Basso E, Petronilli V, Blalchy-Dyson E, Di Lisa F, Forte MA (2006) The mitochondrial permeability transition from in vitro artifact to disease target. Febs J 273(10):2077-2099

15. Brand MD, Nicholls DG (2011) Assessing mitochondrial dysfunction in cells. Biochem J 435(2):297-312

16. Spinazzi M, Casarin A, Pertegato V, Salviati L, Angelini C (2012) Assessment of mitochondrial respiratory chain enzymatic activities on tissues and cultured cells. Nat Protoc 7(6):1235-1246

17. Peters K, Nießen M, Peterhänsel C, Späth B, Hölzle A, Binder S, Marchfelder A, Braun H-P (2012) Complex I-complex II ratio strongly differs in various organs of Arabidopsis thaliana. Plant Mol Biol 79(3):273-284

18. Schägger H, Pfeiffer K (2001) The ratio of oxidative phosphorylation complexes $I-V$ in bovine heart mitochondria and the composition of respiratory chain supercomplexes. J Biol Chem 276(41):37861-37867

\section{Publisher's Note}

Springer Nature remains neutral with regard to jurisdictional claims in published maps and institutional affiliations. 